\title{
Pathway analysis of glioblastoma tissue after preoperative treatment with the EGFR tyrosine kinase inhibitor gefitinib - a phase II trial
}

Hegi, M E ; Diserens, A C ; Bady, P ; Kamoshima, Y ; Kouwenhoven, M C M ; Delorenzi, M ; Lambiv,

W L ; Hamou, M F ; Matter, M S ; Koch, A ; Heppner, F L ; Yonekawa, Y ; Merlo, A ; Frei, K ;

Mariani, L ; Hofer, S

\begin{abstract}
Amplification of the epidermal growth factor receptor (EGFR) gene is one of the most common oncogenic alterations in glioblastoma (45\%) making it a prime target for therapy. However, small molecule inhibitors of the EGFR tyrosine kinase showed disappointing efficacy in clinical trials for glioblastoma. Here we aimed at investigating the molecular effects of the tyrosine kinase inhibitor gefitinib on the EGFR signaling pathway in human glioblastoma. Twenty-two patients selected for reoperation of recurrent glioblastoma were treated within a phase II trial for 5 days with $500 \mathrm{mg}$ gefitinib before surgery followed by postoperative gefitinib until recurrence. Resected glioblastoma tissues exhibited high concentrations of gefitinib (median, $4.1 \mathrm{~g} / \mathrm{g}$ ), 20 times higher than respective plasma. EGFR-pathway activity was evaluated with phosphorylation-specific assays. The EGFR was efficiently dephosphorylated in treated patients as compared to a control cohort of 12 patients. However, no significant effect on 12 pathway constituents was detected. In contrast, in vitro treatment of a glioblastoma cell line, BS-153, with endogenous EGFRwt amplification and EGFRvIII expression resulted not only in dephosphorylation of the EGFR, but also of key regulators in the pathway such as AKT. Treating established xenografts of the same cell line as an in vivo model showed dephosphorylation of the EGFR without affecting downstream signal transductors, similar to the human glioblastoma. Taken together, gefitinib reaches high concentrations in the tumor tissue and efficiently dephosphorylates its target. However, regulation of downstream signal transducers in the EGFR pathway seems to be dominated by regulatory circuits independent of EGFR phosphorylation.
\end{abstract}

DOI: https://doi.org/10.1158/1535-7163.MCT-11-0048

Posted at the Zurich Open Repository and Archive, University of Zurich

ZORA URL: https://doi.org/10.5167/uzh-58617

Journal Article

Accepted Version

Originally published at:

Hegi, M E; Diserens, A C; Bady, P; Kamoshima, Y; Kouwenhoven, M C M; Delorenzi, M; Lambiv, W L; Hamou, M F; Matter, M S; Koch, A; Heppner, F L; Yonekawa, Y; Merlo, A; Frei, K; Mariani, L; Hofer, S (2011). Pathway analysis of glioblastoma tissue after preoperative treatment with the EGFR tyrosine kinase inhibitor gefitinib - a phase II trial. Molecular Cancer Therapeutics, 10(6):1102-1112.

DOI: https://doi.org/10.1158/1535-7163.MCT-11-0048 


\section{Pathway Analysis of Glioblastoma Tissue after Preoperative Treatment with the EGFR Tyrosine Kinase Inhibitor Gefitinib - A Phase II trial}

Monika E Hegi ${ }^{1,2,3}$, Annie-Claire Diserens ${ }^{1,3}$, Pierre Bady ${ }^{3,4}$, Yuta Kamoshima ${ }^{1}$, Mathilde C. M. Kowenhoven ${ }^{5}$, Mauro Delorenzi ${ }^{2,3,4}$, Wanyu L Lambiv ${ }^{1}$, Marie-France Hamou ${ }^{1}$, Matthias S Matter ${ }^{6}$, Arend Koch ${ }^{7}$, Frank L Heppner ${ }^{6,7}$, Yasuhiro Yonekawa ${ }^{8}$, Adrian Merlo $^{9}$, Karl Frei ${ }^{8}$, Luigi Mariani ${ }^{10}$, Silvia Hofer ${ }^{8,11}$

${ }^{1}$ Laboratory of Brain Tumor Biology and Genetics, Service of Neurosurgery, Department of Clinical Neurosciences, Lausanne University Hospital and University of Lausanne, Lausanne Switzerland; ${ }^{2}$ National Center of Competence in Research Molecular Oncology, ISREC-SVEPFL, Lausanne, Switzerland; ${ }^{3} \mathrm{FBM}$ CHUV; ; ${ }^{4}$ Swiss Institute for Bioinformatics, Lausanne, Switzerland; ${ }^{5}$ Erasmus Medical Center, Rotterdam, the Netherlands; ${ }^{6}$ Institute of Neuropathology, University Hospital Zurich, Zurich, Switzerland, ${ }^{7}$ Institute of Neuropathology, Charité-Universitätsmedizin Berlin, Berlin, Germany; ${ }^{8}$ Department of Neurosurgery, University Hospital Zurich; ${ }^{9}$ Laboratory of Molecular Neuro-Oncology, Department of Biomedicine, University Hospital Basel, Basel Switzerland; ${ }^{10}$ Department of Neurosurgery, Inselspital, Bern, Switzerland; ${ }^{11}$ Department of Oncology, University Hospital Zurich

Supported by AstraZeneca (S.H.; M.E.H; M.D.) and OncoSuisse (Grant No. 01680-2 2005; M.E.H.; M. D.)

\section{Running title: EGFR pathway in glioblastoma under gefitinib}

Corresponding Author: Monika E. Hegi, Laboratory of Brain Tumor Biology and Genetics, Department of Neurosurgery, Centre Hospitalier Universitaire Vaudois (CHUV BH19-110), 46, rue du Bugnon, Lausanne 1011, Switzerland Phone: +41-21-314-2582, Fax: +41-21-314-2587, Email: Monika.Hegi@chuv.ch 


\section{Purpose}

Amplification of the epidermal growth factor receptor (EGFR) gene is a common oncogenic alteration in glioblastoma making it a prime target for therapy. However, small molecule inhibitors of the EGFR tyrosine kinase failed in clinical trials for glioblastoma. Here we aimed at investigating the molecular effects of preoperative treatment with the tyrosine kinase inhibitor gefitinib on the EGFR signalling pathway in glioblastoma tissue.

\section{Patients and Methods}

In a phase II trial, 22 patients selected for re-operation of recurrent glioblastoma were treated at least 5 days with $500 \mathrm{mg}$ gefitinib prior to surgery, followed by post-operative gefitinib until recurrence. Resected glioblastoma tissues were tested for drug concentrations and EGFR-pathway activity using phosphorylation specific assays.

\section{Results}

Glioblastoma tissues exhibited high concentrations of gefitinib (median, 4,100 ng/g). The EGFR was efficiently dephosphorylated in treated patients as compared to a control cohort of 12 patients. However, downstream pathway constituents did not seem to be affected. In contrast, in vitro treatment of a glioblastoma cell line, BS-153, with endogenous EGFRwt amplification and EGFRvIII expression resulted not only in dephosphorylation of the EGFR, but also of key regulators in the pathway like AKT. A respective in vivo model, treating established xenografts similar to the human glioblastoma, showed dephosphorylation of the EGFR, without affecting downstream signal transductors.

\section{Conclusion}

Gefitinib reaches high concentrations in the tumor tissue and efficiently dephosphorylates its target. However, regulation of downstream signal transducers in the EGFR pathway seems to be dominated by regulatory circuits independent of EGFR phosphorylation. 


\section{INTRODUCTION}

The epidermal growth factor receptor (EGFR) offers a particularly attractive target in glioblastoma therapy, since it is overexpressed in $60 \%$ of glioblastoma usually associated with high level amplification of the EGFR gene. Small molecule drugs have been developed to specifically target the catalytic kinase domain of the EGFR to prevent downstream signaling ${ }^{1}$. In NSCLC particularly good response was associated with mutations in the EGFR located around the ATP-binding pocket ${ }^{2,3}$. In glioblastoma, however, such mutations have not been found ${ }^{4}$. Some missense mutations have been identified in the extracellular domain of a fraction of cases (14\%) with potentially activating properties ${ }^{5}$, and were usually associated with amplification of the locus. The most common alteration $(20 \%)$, is the truncation mutant lacking exons 2 to 7 (EGFRvIII) affecting the extra cellular domain involved in dimerization and ligand binding that has been associated with constitutive phosphorylation of the receptor conferring an oncogenic potential ${ }^{6,7}$.

A first publication ${ }^{8}$ on a phase II trial testing the EGFR-inhibitor gefitinib in recurrent glioblastoma reported that response to treatment was not correlated with expression of the EGFR, although the authors have not excluded insufficient drug penetration of the tumor. As in most clinical trials for glioblastoma, enzyme inducing antiepileptic drugs (EIAEDs) were allowed in this study that have been shown to reduce systemic availability of the small molecule inhibitors of the EGFR ${ }^{9}$. In the meantime further phase II trials have been reported testing erlotinib or gefitinb in recurrent or progressive glioblastoma, summarized in Yung et al. ${ }^{10}$, or in newly diagnosed glioblastoma as addition to combined chemo-radiotherapy with temozolomide ${ }^{11}$, overall with disappointing efficacy. 
The occasional responses observed in these trials incited several studies to search for predictive molecular markers in the diagnostic tissue of the initial surgery that would allow future patient selection (reviewed in Brandes et al. ${ }^{12}$ ). Several sets of markers with predictive value for response to therapy were proposed, comprising expression of EGFR, amplification of the EGFR gene, lack of elevated levels of AKT phosphorylation, and absence of EGFRvIII expression ${ }^{13}$, while another study suggested better response of tumors with expression of EGFRvIII and expression of PTEN ${ }^{14}$. The markers proposed in these small studies could not be confirmed in subsequent trials, including a randomized phase $\|$ trial ${ }^{9}$, although low p-AKT showed a trend for association with better outcome. The difficulty to successfully target one of the most common activated oncogenic pathways operative in glioblastoma has drastically revealed the complexity of the regulation of receptor tyrosine kinase (RTK) signaling that requires further investigations.

Here we present a phase II clinical trial designed to elucidate potential reasons for the unexpected low response rates of glioblastoma to EGFR inhibitors in previous clinical studies, by addressing the following questions: (i) does the drug reach the tumor across the blood-tumor barrier, (ii) is the EGFR dephosphorylated by the drug, and (iii) what are the effects on downstream signaling. To this end, patients selected for surgery for recurrent glioblastoma were offered participation in a trial with the EGFR inhibitor gefitinib, comprising 5 day pre-operative treatment, followed by postoperative treatment until recurrence or undue side effect. The translational research design included molecular profiling of the human glioblastoma samples obtained from the patients enrolled, and a control set. These efforts were complemented by an experimental in vitro and in vivo model using a tumorigenic glioblastoma cell line with an endogenous EGFR 
amplification and expression of EGFRvIII to aid interpretation of the "noisy" data obtained from human tumors, where no before and after therapy sampling is possible. 


\section{PATIENTS AND METHODS}

Patients selected for re-operation of a recurrent glioblastoma at the University Hospital in Zurich and Bern, Switzerland, were offered to participate in a phase II open label study of pre- and postoperative use of ZD1839 (gefitinib) including translational research (www.clinicaltrials.gov, NCT00250887). Patients were treated at least 5 days with 500 mg gefitinib prior to surgery, followed by post-operative daily use, continuously until tumor progression or occurrence of intolerable side effects. Patients on cytochrome P450 isoenzyme CYP3A4-inducing antiepileptic drugs (EIAEDs) were changed to a nonenzyme-inducing drug, because of predicted ensuing interactions of EIAEDs with gefitinib metabolism, reducing its systemic availability. The trial was approved by the local ethic committees. Resected tumor tissues and plasma samples were snap frozen for further analysis. The following eligibility criteria applied: 18 years and older, fresh frozen sample obtainable, informed consent for translational research; exclusion criteria comprised enzyme inducing antiepileptic drugs. Archived fresh frozen samples of recurrent glioblastoma were used as controls. Patient criteria are summarized in Table 1. All tumor samples underwent central pathology. Frozen tumor tissue was evaluated for tumor content on a Hematoxilin \& Eosin stained frozen section. Samples with a tumor content below $50 \%$ were excluded for molecular analysis.

\section{Drug concentrations}

Frozen tumor tissue and plasma samples were sent to the Laboratory Analytico Medinet B.V. (Breda, NL) to quantify the concentration of gefitinib by high performance liquid chromatography coupled to tandem mass spectrometry as described previously ${ }^{15,16}$. The gefitinib concentrations in the BS-153 xenografts and the mouse plasma were determined at the quantitative Mass Spectrometry Facility (qMSF) at the Lausanne University Hospital using the same technology with minor modifications. The tumor tissue homogenates were prepared at $200 \mathrm{mg}$ of wet weight $/ \mathrm{ml}$ in phosphate buffer using a Fast Prep homogenizer (MP biomedicals, Basel, Switzerland). 


\section{Cell line and xenograft model}

The human glioblastoma cell line BS-153 that comprises an amplified EGFR and expresses the mutant EGFRvIII ${ }^{17}$, was cultured in low serum (0.5\% FCS) and treated with 1,5 and $10 \mu \mathrm{M}$ gefitinib (ZD 1839, AstraZeneca) for $24 \mathrm{~h}$. For in vivo experiments, $10^{7}$ cells were injected subcutaneously into the flanks of immune compromised mice (Swiss nu/nu; Iffa Credo; RCC, BRL). When the tumors reached $1 \mathrm{~cm}$ in diameter the mice were randomized and treated (p.o.) or not with a daily dose of $7 \mathrm{mg} / \mathrm{kg} /$ day gefitinib (suspended in $1 \% \mathrm{w} / \mathrm{v}$ Tween 80 ) by gavage for 5 days that is equivalent to the human dosing scheme of $500 \mathrm{mg} / \mathrm{day}(70 \mathrm{~kg})$. Tumor tissues were harvested $4 \mathrm{~h}$ after the last dose and snap frozen or embedded in paraffin for further analysis. The experiments were approved by the local authorities (protocol VD_1181.3).

\section{Tissue Micro Array, Fluorescence In Situ Hybridization (FISH) and Immunohistochemistry (IHC)}

A tissue micro array (TMA) was constructed from paraffin embedded tumor blocks available from patients and the xenografts in nude mice derived from the human cell line BS-153. FISH for determination of EGFR copy number was performed and scored as described previously ${ }^{9}$. The EGFR copy number was normalized by a centromeric probe on chromosome 12 (CEP12). Immunohistochemical investigations were carried out according to standard procedures for paraffin sections using a heat induced epitope

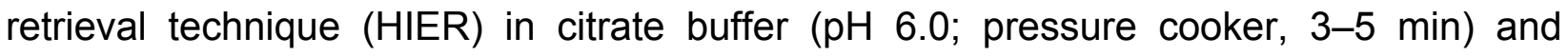
overnight incubation with the primary antibody. The following antibodies were used, $p$ mTOR (1:50, CellSignaling), PTEN (1:50, CellSignaling), CycD (1:50, Upstate). The immunostaining was scored semi-quantitatively (scores, $0-3$ ).

\section{Real-Time Quantitative polymerase chain reaction (qPCR)}

DNA and RNA were isolated from frozen tissue using the Qiagen AllPrep DNA/RNA Kit (Qiagen, 80204). DNA from paraffin sections was isolated as described before ${ }^{18}$. The EGFR gene copy number was determined on DNA isolated from macro-dissected paraffin embedded glioblastoma tissue by the relative $\mathrm{QPCR}$ comparative $\mathrm{Ct}$ ( $2^{\text {-delta delta }}$ 
Ct) method using DNM1L (12p11.21) as reference gene (primer sets: EGFR-F_669 ATGTCCGGGAACACAAAGAC, EGFR-R_670 TTATCTCCCCTCCCCGTATC, amplicon size 104 bp; DNM1L-F_671 TCAGATGTTAAAGCTGCCATTT, DNM1L-R_672 TCCCGAGCAGATAGTTTTCG, amplicon size $101 \mathrm{bp}$ ). Q-PCR was performed on a Rotor Gene 6000 Real-Time PCR system (Corbett Life Science) using the Fast SybR Green Master Mix (Applied Biosystem). DNA from peripheral blood lymphocytes served as normal reference, and cell line BS-153 as positive control for high level amplification. Quantitative reverse transcription PCR (qRT-PCR) was employed for expression analysis of EGFRwt and EGFRvIII at the Genetics Platform at the University of Geneva as described previously ${ }^{19}$.

\section{Western blot and Bio-Plex analysis}

Protein from snap frozen human glioblastoma, xenografts, and cell lines were extracted with the Bio-Plex cell lysis kit (Bio-Rad \# 171-304011) according to manufacturer's protocol. The protein concentration was determined (BCA, Pierce \#23250). Western blot analysis was performed with $20 \mathrm{mg}$ of protein using $7.5 \%$ and $10 \%$ SDS-PAGE gels, and subsequent transfer to a nitrocellulose membrane (Hybond-C, Amersham Life Science). The phosphorylation status of the following proteins was evaluated using antibodies from Cell Signaling: p-EGFR (\#4404), p-AKT (\#9271), p-mTOR (\#2971) pelf4G (\#2441), and p-p90RSK (\#9341) that were revealed by luminescence (BM Chemiluminescence Blotting Substrate, Roche, \#1500694) on films or on a bioluminescence image reader (LAS-4000, Fuji). Tot-Erk1/2 (\#9102) and tubulin (Sigma \# T5168) served as loading controls.

The Multiplexing Bio-Plex total target and phosphoprotein assay (Bio-Rad) was performed at the platform of the Center of Integrated Genomics (CIG) at the University of Lausanne according to Bio-Plex Phosphoprotein detection instruction manual with $0.5 \mathrm{mg} / \mathrm{ml}$ protein in 96 wells (duplicate). The following phospho-proteins were measuerd: pEGFR (pan-phospho), p-AKT $\left(\operatorname{Ser}^{473}\right), p-G S K-3 \alpha / \beta\left(\operatorname{Ser}^{21} / \operatorname{Ser}^{9}\right), p-N F K B$ p65 $\quad\left(\right.$ Ser $\left.^{536}\right), \quad$ p-STAT3 $\quad\left(\operatorname{Tyr}^{705}\right), \quad$ p-ERK1/2 $\quad\left(\operatorname{Thr}^{202} /\right.$ Tyr $^{204}, \operatorname{Thr}^{185} /$ Tyr $\left.^{187}\right), \quad$ pMEK1 $\left(\mathrm{Ser}^{217} / \mathrm{Ser}^{221}\right)$, p-p38MAPK $\left(\mathrm{Thr}^{180} / \mathrm{Tyr}^{182}\right)$, p-p90RSK $\left(\mathrm{Thr}^{359} / \mathrm{Ser}^{363}\right)$, p-p70S6 Kinase $\left(\mathrm{Thr}^{421} / \mathrm{Ser}^{424}\right)$, p-S6 ribosomal Protein $\left(\mathrm{Ser}^{235} / \mathrm{Ser}^{236}\right)$, p-PDGFR-B $\left(\mathrm{Tyr}^{751}\right)$ and 
p-SRC $\left(\mathrm{Tyr}^{416}\right)$. The following total proteins were determined, tot-ERK1/2 (\#171V32238), tot-p38MAPK (\#171-V31336), tot-EGFR, tot-MEK1, and tot-AKT. Tot-ERK1/2 was present on all plates and was used for normalization of the data-set after $\log _{2}$ transformation.

\section{Statistical Methods}

Statistical significance of differences between treatments was evaluated with a nonparametric test, Wilcoxon's test and the difference between subgroups was tested by Kruskal \& Wallis' test. The independence between the two variables was tested by exact Fisher's test. A Monte-Carlo test on between-group inertia (global test) was performed to test the overall difference between treated and untreated patients ${ }^{20}$. The heatmap representations were obtained with the Ward's algorithm using euclidean distance. The data was scaled and centered by phospho-proteins if not stated differently. All analyses and graphical representation were done in $\mathrm{R}^{21}$ (URL http://www.R-project.org ) and the $\mathrm{R}$ packages gplots and ade ${ }^{22}$. 


\section{RESULTS}

Twenty-two patients with recurrent glioblastoma selected for second surgery were enrolled between May 2005 and July 2007. Patients were treated for a median of 7.5 days (range 5 to 150 days) with 500 mg gefitinib daily prior to surgery, followed by postoperative gefitinib until recurrence. The study outline is depicted in Fig. S1. Central review confirmed recurrent glioblastoma in all cases. For three cases no frozen tissue became available, and several cases had to be excluded for molecular analysis, because the available frozen tissue consisted of $>80 \%$ necroses or showed only reactive changes. Samples considered for further analysis had to display $>50 \%$ compact viable tumor tissue (Table 1).

\section{EGFR amplification status}

EGFR amplification was identified in 7 of 22 (32\%) patients in the gefitinib group and 7 of $12(58 \%)$ patient samples of the control group, as determined by FISH on the TMA and/or by qPCR from whole tumor sections (Table 1, Fig.1). A good correlation of the copy number was observed between both technologies $\left(r_{\text {pearson }}=0.87\right.$ and $r_{\text {spearman }}=0.83$ ). For three patients we had to infer the EGFR status from the analysis of the glioblastoma tissue from the first resection, as the tissues obtained at re-operation did not comprise enough tumor cells. Overexpression of the wild-type EGFR was associated with amplification of the EGFR gene, but no linear correlation was observed. Expression of the EGFRVIII was detectabled in two glioblastoma with an amplified EGFR status. (Fig. 1)

\section{Gefitinib concentrations in tumor tissue and plasma}

The median concentration of gefitinib in the tumor tissue was 4,100 ng/g (range 16 to $26,000 \mathrm{ng} / \mathrm{g}$ ), while the respective median concentration in the plasma was more than $20 x$ lower $181 \mathrm{ng} / \mathrm{ml}$ ( 4 to $483 \mathrm{ng} / \mathrm{ml}$ ) ( $p<0.0001)$. The median time laps between the last drug dose and collection of the tumor tissue and the blood sample was $5 \mathrm{~h}$ and 5 h $30 \mathrm{~min}$, respectively. One patient did not take the drug on the day of surgery, reflected in very low drug concentrations in the tumor and the blood, respectively (Fig. 2). Timed information was only recorded in one center, however, drug concentrations suggested a 
similar timeframe for tissue and blood collection for the other patients. The mean gefitinib concentration in the mouse BS-153 xenografts $(n=9)$ was 1,669 ng/g (range, 457 to $3,620 \mathrm{ng} / \mathrm{g}$ ) and $226 \mathrm{ng} / \mathrm{ml}$ (range, $65-336 \mathrm{ng} / \mathrm{ml}$ ) in the serum $(\mathrm{n}=4)$. The tumors were harvested $4 \mathrm{~h}$ after the last gavage of drug.

\section{Patient outcome}

The median survival after initiation of gefitinib treatment was 8.8 months. No difference was observed between patients with an amplified or a normal EGFR status. However, patients whose resected tissue had to be excluded for molecular analysis due to predominantly necrotic tissue had longer survival (logrank, $\mathrm{P}=0.004$ ). This may be an indication of pseudo-progression. However, the small patient numbers preclude proper analysis and interpretation of this observation.

\section{Molecular analysis of the EGFR signalling pathway in glioblastoma}

In order to investigate the effect of gefitinib on activation of the EGFR and respective downstream signaling, a phosphorylation screen for a selected panel of 12 published EGFR pathway signal transductors was performed. Beside pEGFR, it included constituents such as pAKT, pERK1/2 or pS6RP to cover distinct aspects of the pathway. Further, it included PDGFR-B and SRC two important players in glioblastoma. PDGFR-B activates elements of the same pathway, while SRC has to been reported to be an effector of EGFR signaling ${ }^{23}$. Comparison between treated and untreated patients revealed that there was a border line significant decrease of phosphorylation of the EGFR ( $p=0.053)$, and surprisingly an increase of $p$-ERK1/2 phosphorylation $(p=0.076)$. The phosphorylation of the other signaling transductors were not significantly changed. When stratifying for the EGFR amplification status, there was a significant reduction of EGFR phosphorylation $(p=0.006)$, indicating efficient dephosphorylation by gefitinib as indicated by box plots in Figure 3A. Further, a trend for increased phosphorylation of ERK1/2 $(p=0.104)$ was observed (Fig. 3B). The statistical analysis for all phosphoproteins is summarized in Table $\mathrm{S} 1$ and the respective box plots are displayed in Fig. S2. The overall difference between treated and untreated patient samples did not reach statistical significance ( $p=0.139$, Monte-Carlo test, 999 permutations, Fig. $3 C)$, thus the 
gefitinib treatment effect seems to be mostly restricted to dephosphorylation of the EGFR. Exclusion of 4 cases from the analysis who did not adhere strictly to the protocol, such as extended pre-treatment period ( 3 cases), or missed drug intake on the day of surgery ( 1 case) did not reveal other significant factors.

An interaction map of the pathway, indicating the analyzeded proteins, and a heatmap of the phosphorylation profiles are shown in Fig. 4. The dendrogram from this unsupervised analysis suggests that EGFR signaling was not dominating the activity of the pathway. In fact EGFR phosphorylation was least related to pathway activation as indicated by principal component analysis (PCA) of all measured phospho-proteins (Fig. 4C). The closest correlation was with pPDGFR-B (Pearson correlation $r=0.5$ ) (Fig. S3, shows all pairwise comparisons), however, the amplitude of pPDGFR-B was much lower (Fig. S3,4). Information on expression of PTEN, CycD and p-mTOR was obtained by immunohistochemistry on the respective TMA, included as label to the heatmap (Fig. $4 B)$.

\section{Effect of gefitinib on EGFR signaling pathway in an in vitro and in vivo model}

In parallel to the human clinical trial we investigated gefitinib modulation of EGFR signaling in an in vitro and in vivo model to facilitate interpretation of the human data. The human glioblastoma cell line BS-153, with inherent amplification and overexpression of the EGFR and overexpression of the mutant EGFRVIII (Fig 1,5), was subjected to gefitinib treatment in vitro and in vivo. In vitro experiments carried out over $24 \mathrm{~h}$ showed as expected dephosphorylation of the EGFRwt\&vIII, and also reduced phosphorylation of key signal transductors, such as AKT that is involved in cell survival signaling, and p90RSK a regulator of cell growth and differentiation (Fig. 5). Treatment of mice with established subcutaneous BS-153 xenografts according to the human gefitinib dosing schedule also resulted in efficient dephosphorylation of the EGFRwt\&vIll. However, in contrast to the in vitro experiments phosphorylation of downstream signal transductors were not modulated. Hence, in the in vivo setting the results are similar to those obtained from the human glioblastoma samples. Interestingly, mTOR and elF4G that are involved in nutrition sensing and regulation of protein translation, respectively, were generally less activated in the BS-153 xenograft tissues 
as compared to the cell lines in vitro. This may indicate differences of metabolism in the two model systems. Modulation of mTOR and elF4G phosphorylation was observed in vitro upon stimulation with EGF or treatment with gefitinib (Fig 5).

\section{DISCUSSION}

Small molecule inhibitors of the EGFR have shown little activity in glioblastoma despite the fact that this pathway is affected through amplification of the EGFR gene in around $50 \%$ of patients. The present phase II trial was aimed at elucidating gefitinib mediated modulation of known EGFR downstream signaling. Patients were moved onto non-EIAEDs before study entry, to exclude reduced drug exposure through induction of CYP3A4. Intra-tumoral gefitinib concentrations reached high levels that were 20-times higher than the respective plasma concentrations, when measured within $8 \mathrm{~h}$ from the last drug intake. Most importantly, gefitinib treatment was associated with efficient dephosphorylation of the EGFR. This is in contrast to tumor drug concentrations reported for erlotinib or its active metabolite (OSI-420) that were low, and reaching only 6 to $50 \%$ of the respective plasma concentrations ${ }^{24,25}$. It may thus not be surprising that the authors reported inconsistent EGFR dephosphorylation in the respective tumor tissues ${ }^{24,25}$.

Despite the efficient EGFR dephosphorylation by gefitinib, a phospho-screen of signal transducers downstream of EGFR did not show a statistically significant modulatory effect on the pathway, although a trend for increased phosphorylation of ERK1/2 was observed (Table S1). This is counterintuitive, since a decrease if anything would have been expected according to the interaction map of the pathway. The overall inertness of the pathway signal transductors to EGFR dephosphorylation by gefitinib may not surprise given our observation that EGFR phosphorylation was not indicative of overall activation of the pathway regardless of the treatment (Fig. 4C). It has been proposed that the signaling network, constituted by the ERBB family of receptors of which EGFR is a member (ERBB1), and other mitogenic receptors involved in the malignant behavior of glioblastoma such as MET, or PDGFR, is very robust, since it shares modularity (parts of the pathway), and shows redundancy of regulatory circuits 
26,27. Interestingly, an in vivo model treating established human tumor xenografts with endogenous amplification and overexpression of the EGFRwt \& vIII, recapitulated the efficient EGFR dephosphorylation by gefitinib in glioblastoma, and reproduced lack of downstream signaling modulation. In contrast, treatment of the same cell line in vitro modulated phosphorylation of the signal transductors, thus failing to predict in vivo behavior. Reasons comprise the fact that in vitro experiments usually model acute exposure (here $24 \mathrm{~h}$ ), while treatment in glioblastoma and in vivo models may allow escape through adaptive changes utilizing the redundancy of the regulatory circuits. Moreover, respective analysis at resection likely shows a snapshot of a newly established steady state. In addition, in vitro systems lack stress signaling induced in vivo by metabolic stress, or hypoxia that share some of the downstream signal transductors.

In conclusion, the present study suggests that the EGFR inhibitor gefitinib reaches the tumor in high concentrations, efficiently dephosphorylates the target, however, this does not suffice to control pathway activity. EGFR-phosphorylation independent regulatory circuits seem to dominate the pathway. In order to find therapeutic opportunities, the fragilities of the network need to be enquired that may help to design promising combination therapies for patients with respective molecular characteristics ${ }^{26,28}$. 


\title{
Acknowledgements:
}

We are indebted to patients and their families to allow translational research on tumor tissue. We thank our colleagues in Neurosurgery for providing fresh tumor tissue, Drs Migliavacca, and Schutz for data preparation, and Drs Rochat, Murat, Descombes, Chollet, and Talbot for excellent technical support.

\section{AUTHOR'S DISCLOSURES OF POTENTIAL CONFLICTS OF INTEREST}

\author{
Employment or Leadership Position: None \\ Consultant or Advisory Role: None \\ Stock Ownership: None \\ Honoraria: None \\ Research Funding: Monika E. Hegi, AstraZeneca; Silvia Hofer, AstraZeneca; Luigi \\ Mariani, AstraZeneca \\ Expert Testimony: None \\ Other Remuneration: None
}

\section{AUTHOR CONTRIBUTIONS}

Conception and design: Monika Hegi, Silvia Hofer

Financial support: Monika E. Hegi, Silvia Hofer

Administrative support: Silvia Hofer, Monika Hegi

Provision of study materials or patients: Silvia Hofer, Annie-Claire Diserens, Yuta Kamoshima, Mathilde Kowenhoven, Monika E. Hegi, Luigi Mariani, Matthias Matter, Frank Heppner, Arend Koch, Adrian Merlo, Karl Frei, Yasuhiro Yonekawa, Marie-France Hamou, Wanyu Lambiv

Data analysis and interpretation: Monika Hegi E., Pierre Bady, Silvia Hofer Manuscript writing: Monika Hegi, Silvia Hofer, Pierre Bady

Final approval of manuscript: Silvia Hofer, Monika E. Hegi, Pierre Bady, Annie-Claire Diserens, Yuta Kamoshima, Mathilde Kowenhoven, Luigi Mariani, Matthias Matter, Frank Heppner, Arend Koch, Adrian Merlo, Karl Frei, Yasuhiro Yonekawa, Marie-France Hamou, Wanyu Lambiv, Mauro Delorenzi 


\section{REFERENCES}

1. Mendelsohn J, Baselga J: The EGF receptor family as targets for cancer therapy. Oncogene 19:6550-6565., 2000

2. Lynch TJ, Bell DW, Sordella R, et al: Activating Mutations in the Epidermal Growth Factor Receptor Underlying Responsiveness of Non-Small-Cell Lung Cancer to Gefitinib. N Engl J Med 350:2129-39, 2004

3. Paez JG, Janne PA, Lee JC, et al: EGFR Mutations in Lung Cancer: Correlation with Clinical Response to Gefitinib Therapy. Science 304:1497-1500, 2004

4. Frederick L, Wang $X Y$, Eley $G$, et al: Diversity and frequency of epidermal growth factor receptor mutations in human glioblastomas. Cancer Res 60:1383-7., 2000

5. Lee JC, Vivanco I, Beroukhim R, et al: Epidermal Growth Factor Receptor Activation in Glioblastoma through Novel Missense Mutations in the Extracellular Domain. PLoS Med 3:e485, 2006

6. Ekstrand AJ, James CD, Cavenee WK, et al: Genes for epidermal growth factor receptor, transforming growth factor a, and epidermal growth factor and their expression in human gliomas in vivo. Cancer Res 51:2164-2172, 1991

7. Nishikawa R, Ji XD, Harmon RC, et al: A mutant epidermal growth factor receptor common in human glioma confers enhanced tumorigenicity. Proc Natl Acad Sci U S A 91:7727-31., 1994

8. Rich JN, Reardon DA, Peery $\mathrm{T}$, et al: Phase II trial of gefitinib in recurrent glioblastoma. J Clin Oncol 22:133-142., 2004

9. van den Bent MJ, Brandes AA, Rampling R, et al: Randomized phase II trial of erlotinib versus temozolomide or carmustine in recurrent glioblastoma: EORTC brain tumor group study 26034. J Clin Oncol 27:1268-74, 2009

10. Yung WK, Vredenburgh JJ, Cloughesy TF, et al: Safety and efficacy of erlotinib in first-relapse glioblastoma: a phase II open-label study. Neuro Oncol 2010:8, 2010 11. Peereboom DM, Shepard DR, Ahluwalia MS, et al: Phase II trial of erlotinib with temozolomide and radiation in patients with newly diagnosed glioblastoma multiforme. $\mathrm{J}$ Neurooncol 4:4, 2009 
12. Brandes A, Franceschi E, Tosoni A, et al: Epidermal Growth Factor Receptor Inhibitors in Neuro-oncology: Hopes and Disappointments. Clin Cancer Res. 14:957-60., 2008

13. Haas-Kogan DA, Prados MD, Tihan T, et al: Epidermal growth factor receptor, protein kinase B/Akt, and glioma response to erlotinib. J Natl Cancer Inst 97:880-7., 2005

14. Mellinghoff IK, Wang MY, Vivanco I, et al: Molecular determinants of the response of glioblastomas to EGFR kinase inhibitors. N Engl J Med 353:2012-24, 2005

15. Zhao M, Hartke C, Jimeno A, et al: Specific method for determination of gefitinib in human plasma, mouse plasma and tissues using high performance liquid chromatography coupled to tandem mass spectrometry. J Chromatogr B Analyt Technol Biomed Life Sci 819:73-80, 2005

16. Hofer S, Frei K: Gefitinib concentrations in human glioblastoma tissue. J Neurooncol 82:175-6, 2007

17. Jones G, Machado J, Jr., Merlo A: Loss of focal adhesion kinase (FAK) inhibits epidermal growth factor receptor-dependent migration and induces aggregation of $\mathrm{nh}(2)-$ terminal FAK in the nuclei of apoptotic glioblastoma cells. Cancer Res 61:4978-81., 2001

18. Vlassenbroeck I, Califice $S$, Diserens $A C$, et al: Validation of real-time methylation-specific PCR to determine O6-methylguanine-DNA methyltransferase gene promoter methylation in glioma. J Mol Diagn 10:332-7, 2008

19. Murat A, Migliavacca E, Gorlia T, et al: Stem cell-related "self-renewal" signature and high epidermal growth factor receptor expression associated with resistance to concomitant chemoradiotherapy in glioblastoma. J Clin Oncol 26:3015-24, 2008

20. Romesburg HC: Exploring, confirming, and randomization tests. Computers \& Geosciences 11:19-37, 1985

21. R Development Core Team: R: A language and environment for statistical computing. Vienna, Austria., R Foundation for Statistical Computing, 2010

22. Chessel D, Dufour AB, Thioulouse J: The ade4 package-I- One-table methods. $R$ News 4:5-10, 2004 
23. Lu KV, Zhu S, Cvrljevic A, et al: Fyn and SRC are effectors of oncogenic epidermal growth factor receptor signaling in glioblastoma patients. Cancer Res 69:6889-98, 2009

24. Lassman AB, Rossi MR, Razier JR, et al: Molecular study of malignant gliomas treated with epidermal growth factor receptor inhibitors: tissue analysis from north american brain tumor consortium trials 01-03 and 00-01. Clin Cancer Res 11:7841-50, 2005

25. Raizer JJ, Abrey LE, Lassman AB, et al: A phase II trial of erlotinib in patients with recurrent malignant gliomas and nonprogressive glioblastoma multiforme postradiation therapy. Neuro Oncol 12:95-103, 2009

26. Citri A, Yarden Y: EGF-ERBB signalling: towards the systems level. Nat Rev Mol Cell Biol 7:505-16, 2006

27. Amit I, Wides R, Yarden Y: Evolvable signaling networks of receptor tyrosine kinases: relevance of robustness to malignancy and to cancer therapy. Mol Syst Biol 3:151, 2007

28. Stommel JM, Kimmelman AC, Ying $\mathrm{H}$, et al: Coactivation of receptor tyrosine kinases affects the response of tumor cells to targeted therapies. Science 318:287-90, 2007

29. Bertotti A, Burbridge MF, Gastaldi S, et al: Only a subset of met-activated pathways are required to sustain oncogene addiction. Sci Signal 2:er11, 2009 


\section{Figure Legends}

Figure 1. EGFR amplification and expression in glioblastoma. EGFR copy number corresponding to the maximum value determined by FISH on the TMA or qPCR on whole tissue sections are represented by black lines (left scale). Expression of EGFRwt (filled triangle, red) and EGFRvIII (open triangle, red) was determined by qRT-PCR, the scale on the right hand side applies. The samples called 'xeno0' and 'xeno1' indicate the averaged values for the untreated and treated BS-153 xenografts in mice, respectively. EGFRvIII expression was detected in two glioblastoma at modest levels not exceeding expression of EGFRwt. BS-153 xenografts displayed high expression of EGFRvIII greater than EGFRwt.

Figure 2. Gefitinib concentrations in the tumor tissue and plasma.

Gefitinib concentrations were measured in the tumor tissue and plasma obtained after a median time laps of $5 \mathrm{~h}$ and $5 \mathrm{~h} 30 \mathrm{~min}$ after the last gefitinib intake, respectively. The median gefitinib concentration in the tumor tissue was 20-times higher than in the plasma $(4,100 \mathrm{ng} / \mathrm{g}$, range 16 to $26,000 \mathrm{ng} / \mathrm{g} ; 181 \mathrm{ng} / \mathrm{ml}$, range 4 to $483 \mathrm{ng} / \mathrm{ml}$; $p<0.0001)$. One patient did not take the drug on the day of surgery $(>24 \mathrm{~h})$, reflected in very low drug concentrations in the tumor and the plasma, respectively.

Figure 3. Effect of gefitinib on EGFR pathway signaling transductors. Fourteen signaling transductors of the EGFR pathway were determinded by Bio-Plex technology and normalized to tot-ERK1/2. The log2-intensity of the p-EGFR (A) and p-ERK1/2 (B) measured in the tumor tissues from patients under gefitinib treatment $(\mathrm{T} 1$, red) or a control cohort (T0, blue) are represented in box plots stratified by the EGFR 
amplification status (A1, amplified; A0, not amplified). A significant decrease was found for pEGFR ( $p=0.006$, Kruskal \& Wallis test), and a trend for higher $p E R K 1 / 2(p=0.1)$. Overall differences over the 14 measured phospho-proteins between tumors under gefitinib treatment and controls are not significant (C). Box plots for the other 12 phospho-proteins are available in Fig. S2.

Figure 4. Modulation of the EGFR signaling pathway. (A) The EGFR pathway interaction map is adapted from Bertotti et al. ${ }^{29}$ and indicates the phospho-proteins measured by Bio-Plex analysis in pink, and proteins determined by immunohistochemistry on the TMA or by Western in yellow. (B) The heatmap clusters the samples and phospho-proteins by similarity. Tumors with EGFR amplification are marked in green (A1; no amplification A0, light green). Tumors under gefitinib treatment are indicated in red (T1), and blue for the controls (T0). For one patient with an amplified EGFR a sample at second relapse was obtained and showed high p-EGFR (ZH-06b) in contrast to the tumor under gefitinib treatment ( $\mathrm{ZH}-06 \mathrm{a})$. Sample ${ }^{*} 2510$ is the recurrent glioblastoma of ${ }^{*} 2505$. A heatmap without scaling and centering is available as Fig. S4 that visualizes the differences in amplitude of the different phospho-proteins. The pathway constituents PTEN, CycD, and mTOR were evaluated semi-quantitatively by immunhistochemistry and have been added as labels (blue, no expression; grades of pink, increasing expression 1-3; white, no information; for mTOR, 0/vs, tumor negative vessels positive, dark blue). The dendrogram of the phospho-proteins indicates that $p$ EGFR is very distant to the other pathway signaling transductors. (C) The Principal component analysis (PCA) of the phospho-proteins shows the first vectorial plan based on the correlation matrix. The first axis of the PCA represented $62 \%$ of the variance 
(total inertia) of the table and organized the phospho-proteins in function of their total intensity. All phospho-proteins were turned in the same sense, with the exception of pEGFR and to a lesser extent p-PDGFR-B. The second axis was mainly built by the variable p-EGFR and it explained $9.2 \%$ of the variance of all phospho-proteins. On the first vectorial plan of the PCA, we observe that the expression of $p$-EGFR is not correlated with the other phospho-proteins, except for $p$-PDGFR-B ( $r=0.527)$. Pairwise correlations of all phospho-proteins are displayed in a matrix of scatter plots in Fig. S3.

Figure 5. Modulation of the EGFR pathway by gefitinib in vitro and in vivo. The human glioblastoma cell line BS-153 was treated in vitro with EGF or 1 and $5 \mu \mathrm{M}$ gefitinib for 24 h. Of note, $5 \mu \mathrm{M}$ gefitinib already had some toxic effects on the cells. Nude mice with established subcutaneous BS-153 xenografts (diameter ca. $1 \mathrm{~cm}$ ) were treated 5 days with gefitinib according to the human schedule. The activation status of the EGFR pathway was measured in all protein extracts by Bio-Plex technology in parallel with the human glioblastoma tissues (Fig. 4). (A) A heatmap for the phospho-proteins normalized by tot-ERK $1 / 2$ is shown (without scaling and centering). In the xenografts gefitinib treatment reduced pEGFR, while in the in vitro experiment several signaling transductors were modulated including p-AKT. Three of the phospho-proteins are also shown by Western analysis (B), confirming the results obtained by Bio-Plex analysis. In addition, p-mTOR and p-elF4G were measured, indicating that these signal transductors of metabolism sensing and protein translation were generally lower in the xenografts as opposed to the in vitro model. 
Table 1. Baseline Patient Characteristics

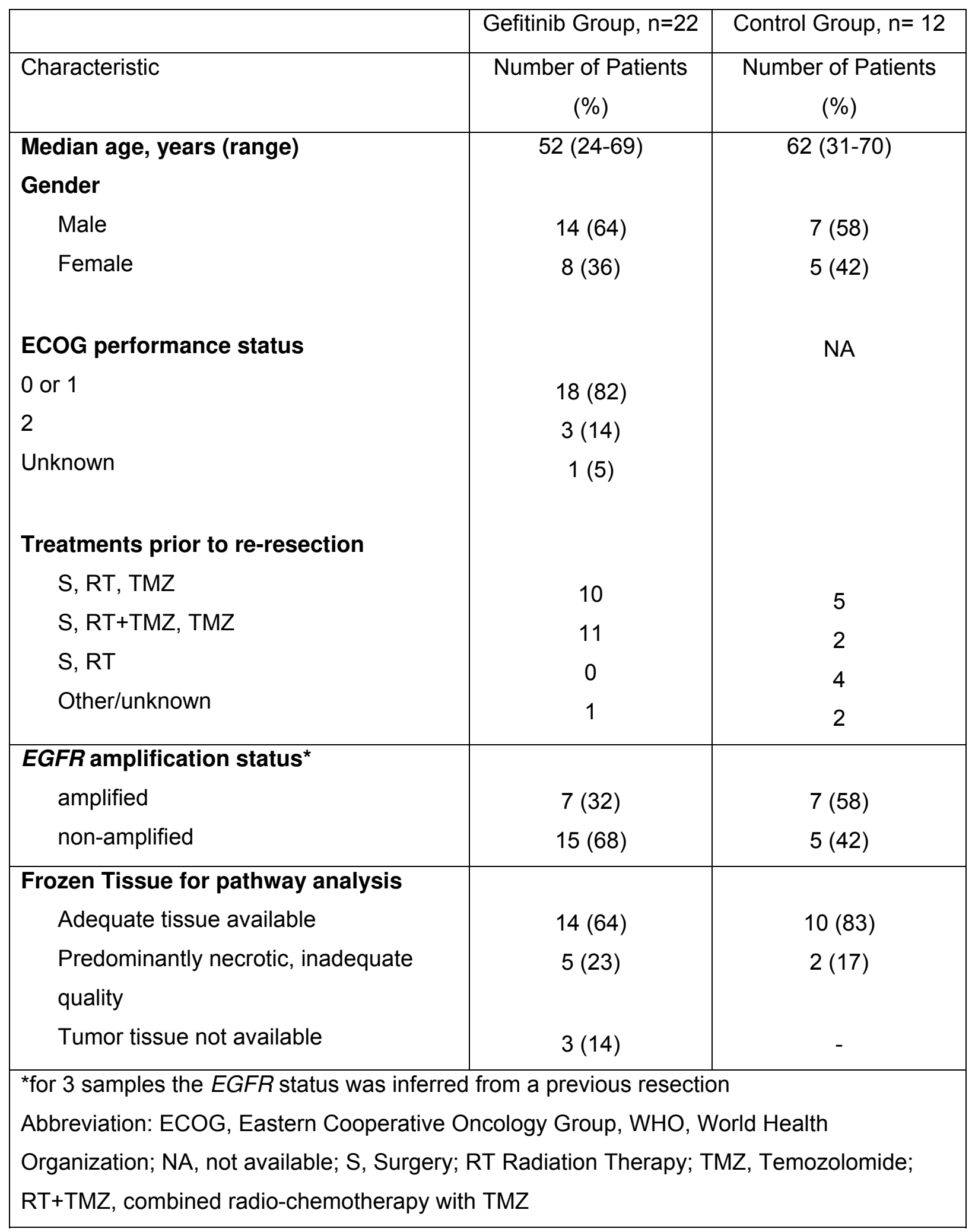




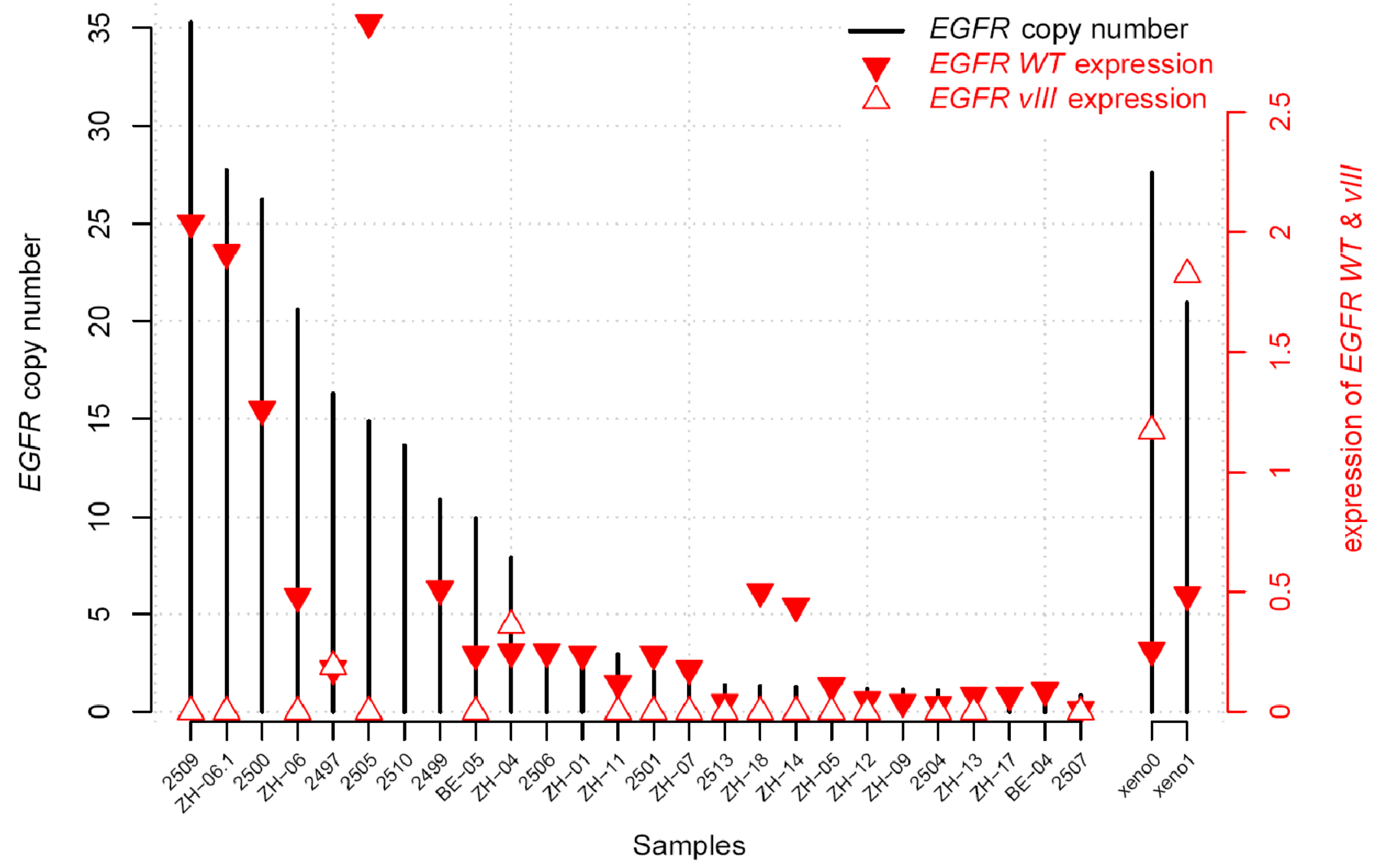

Fig. 1 


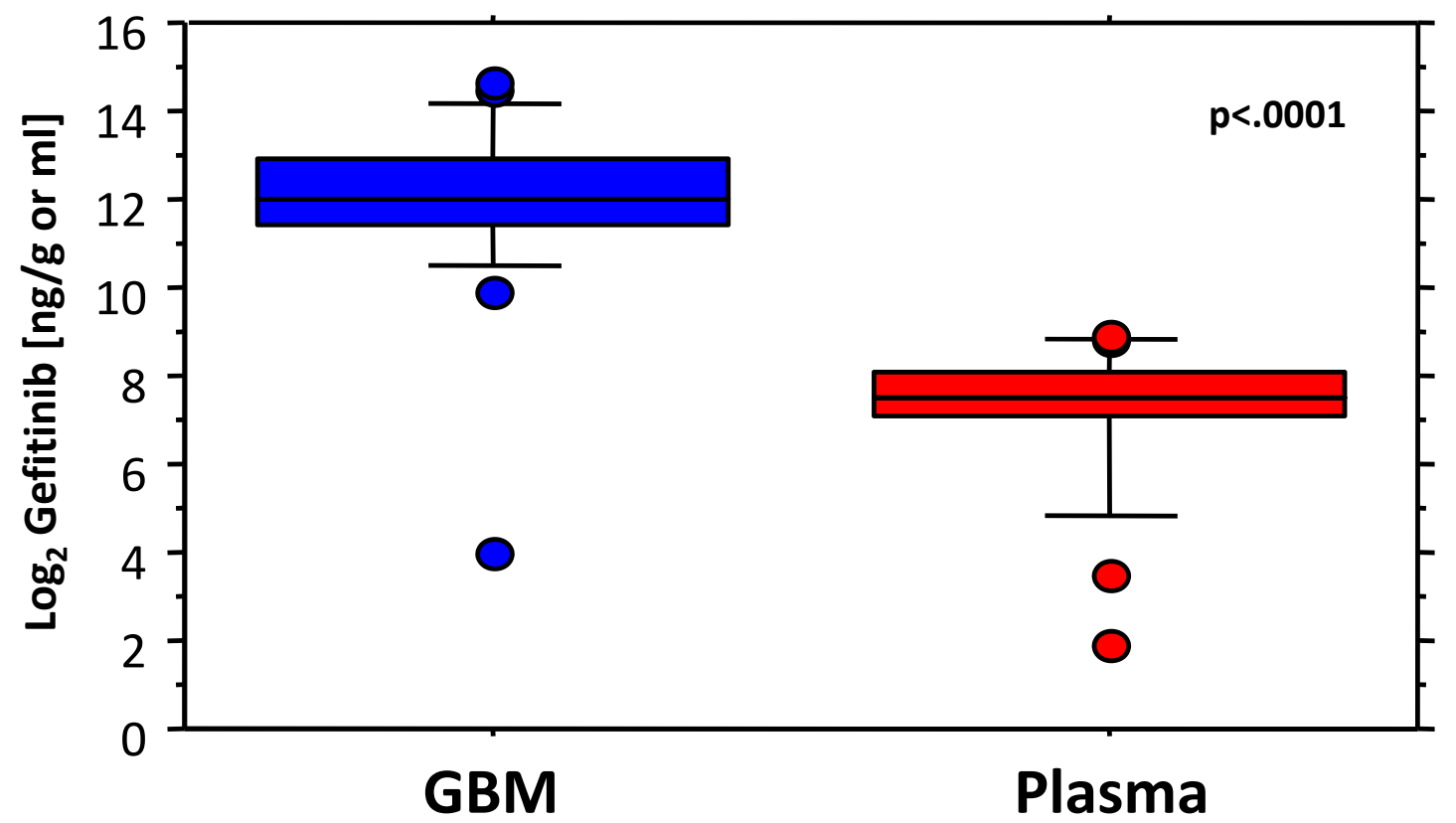

Fig. 2 


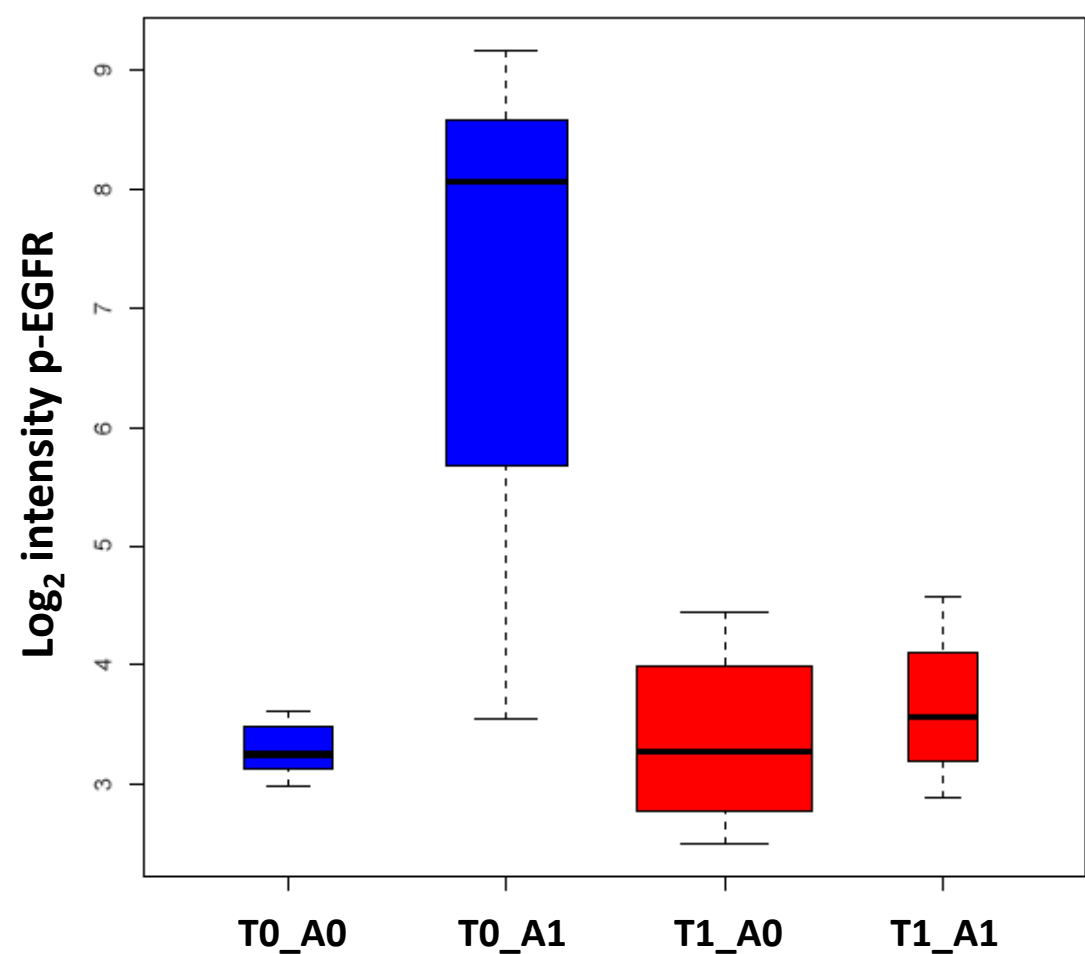

A

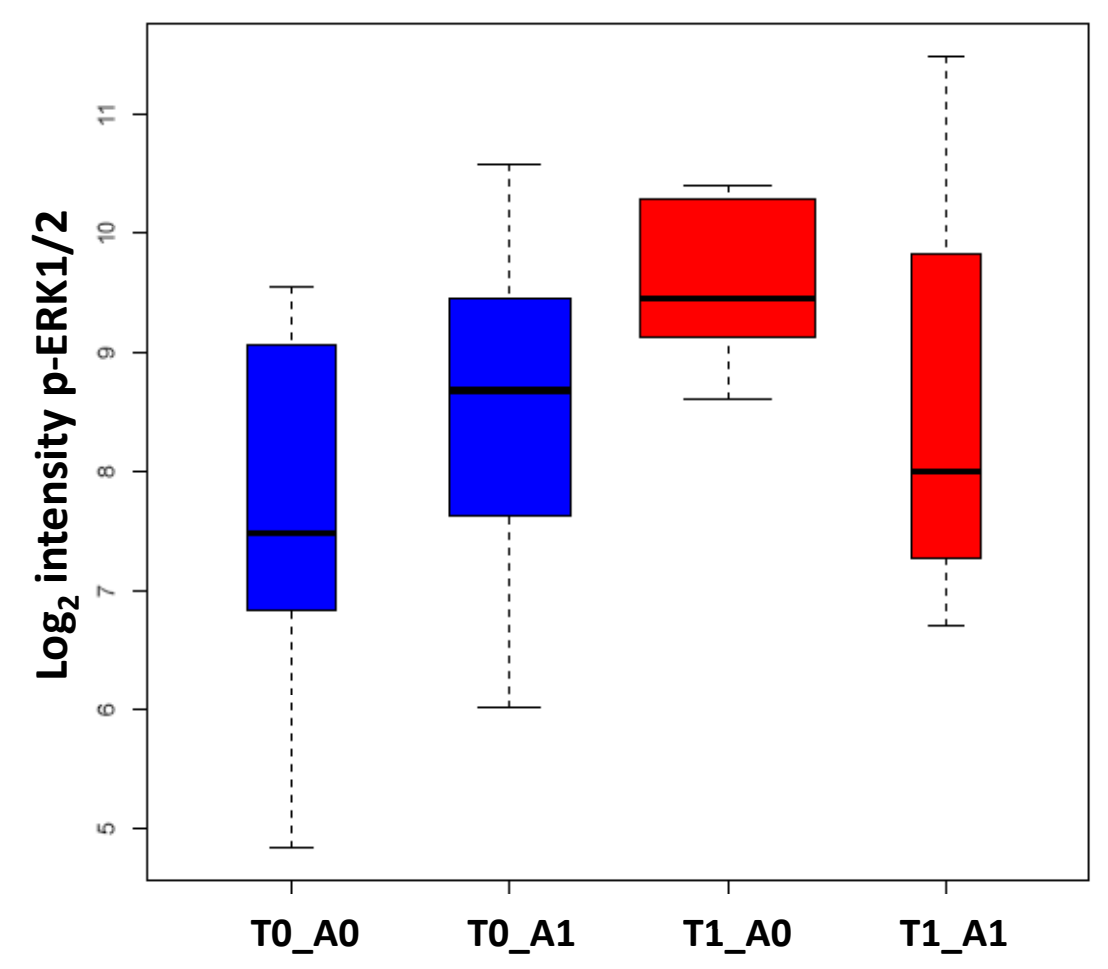

B

Fig. 3 


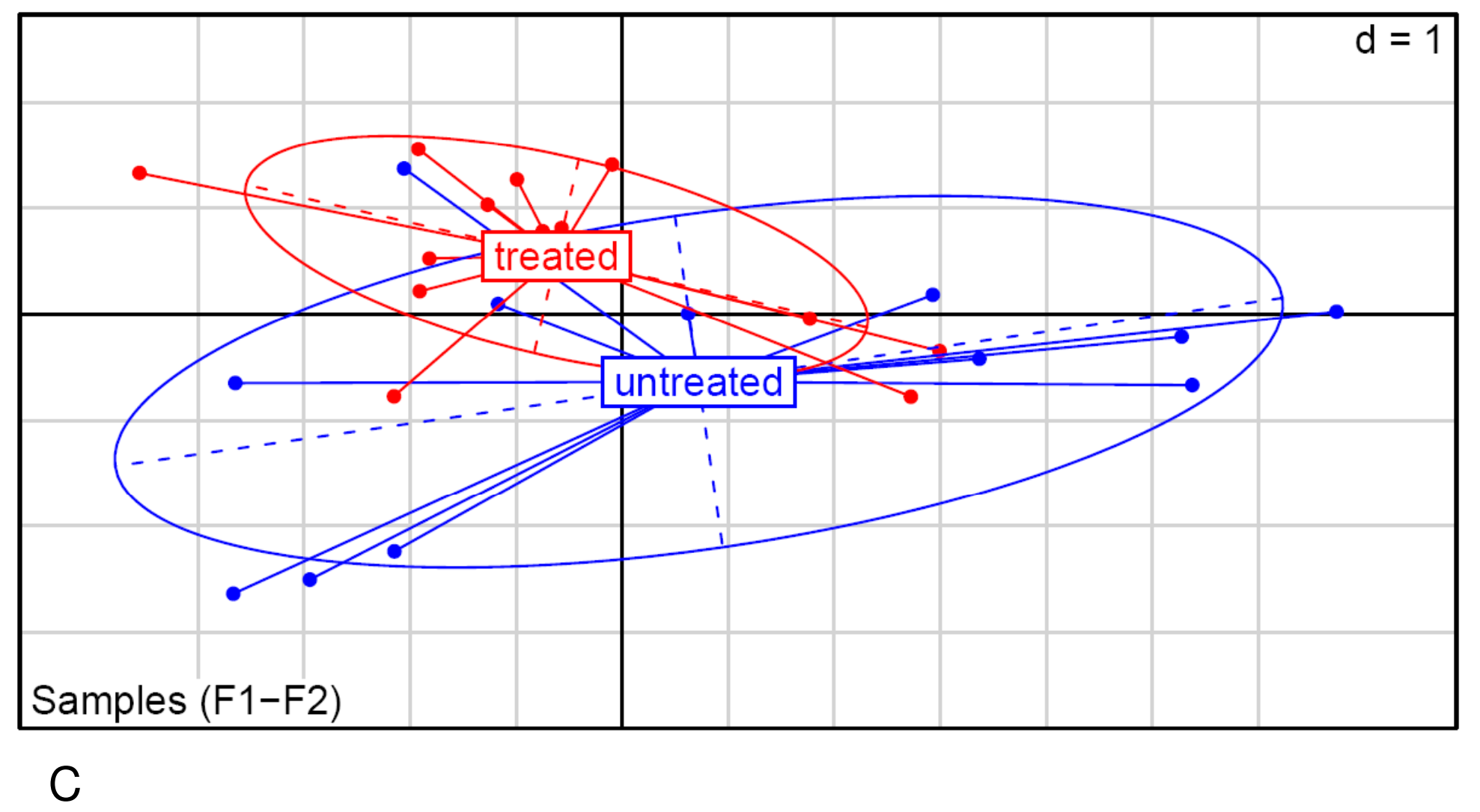

Fig. 3C 


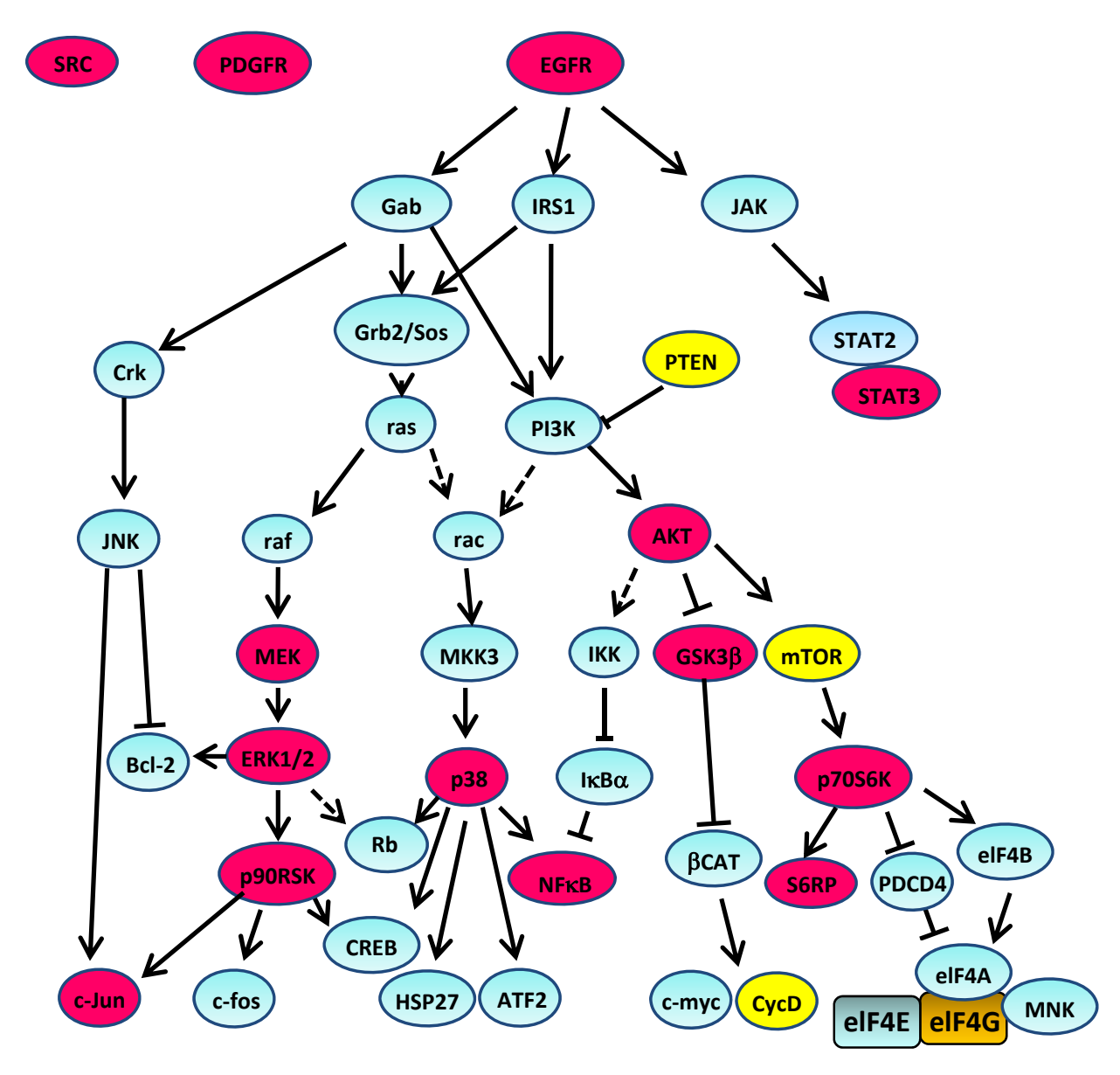

Fig. 4A 


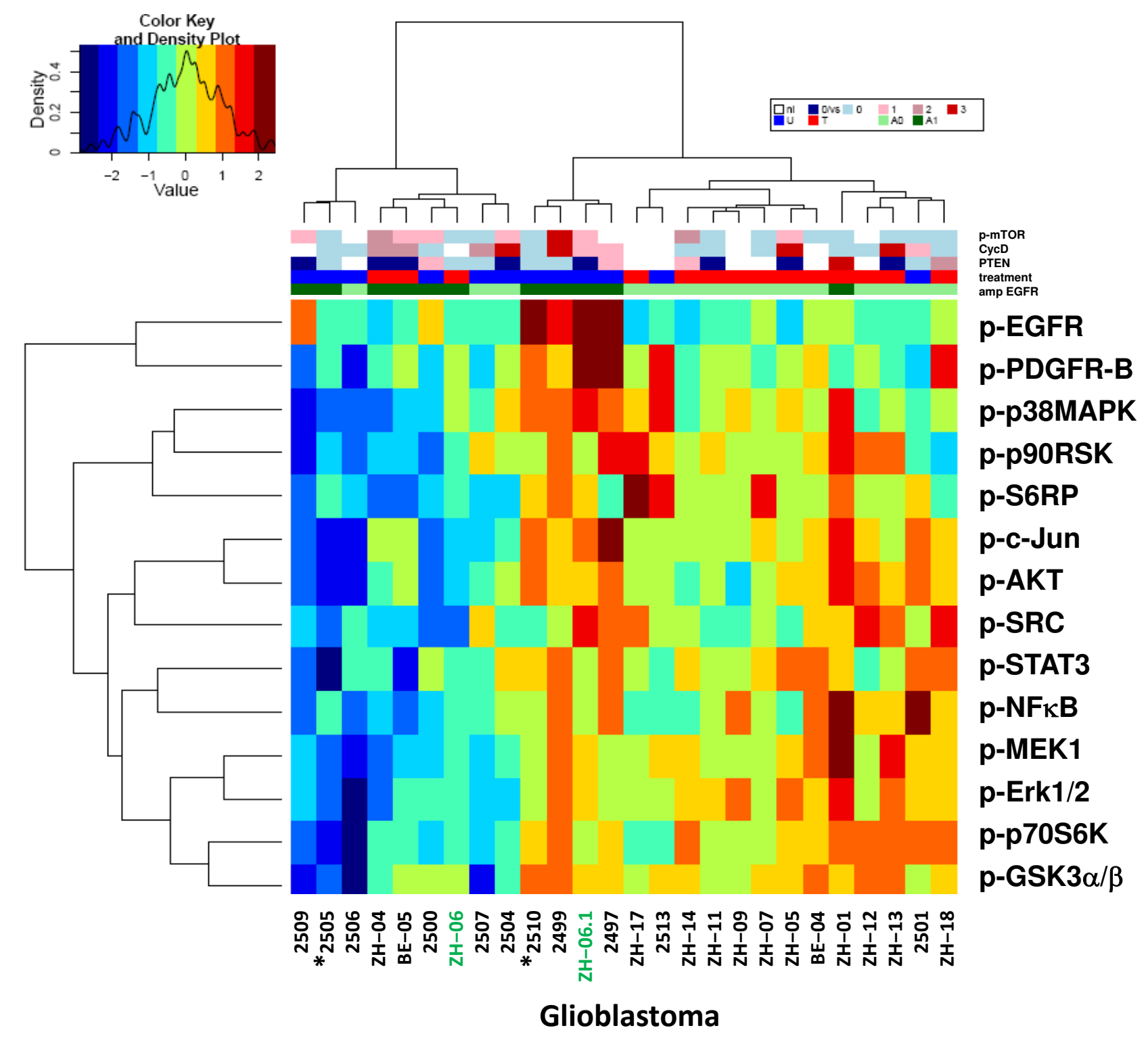

Fig. 4B 


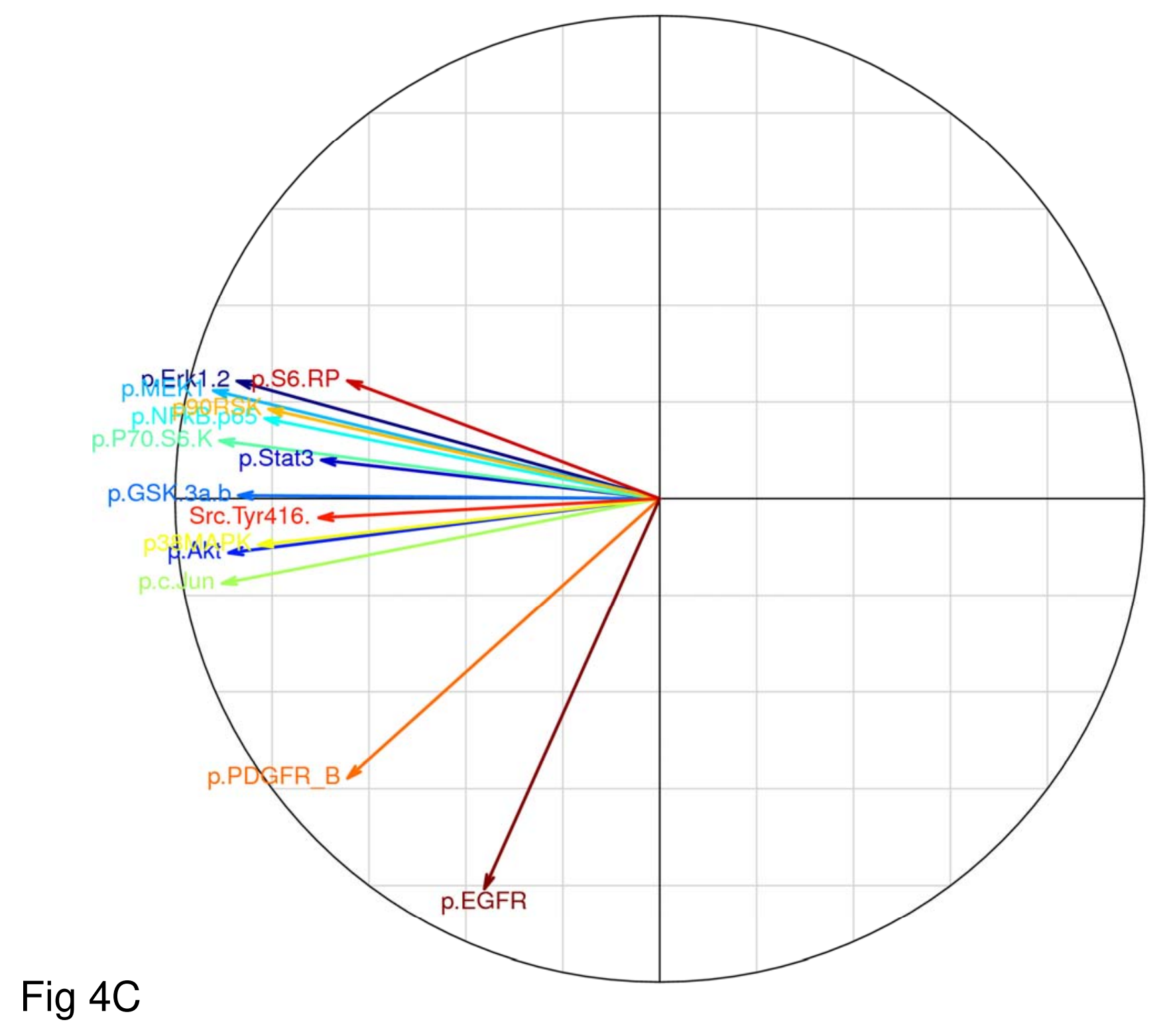




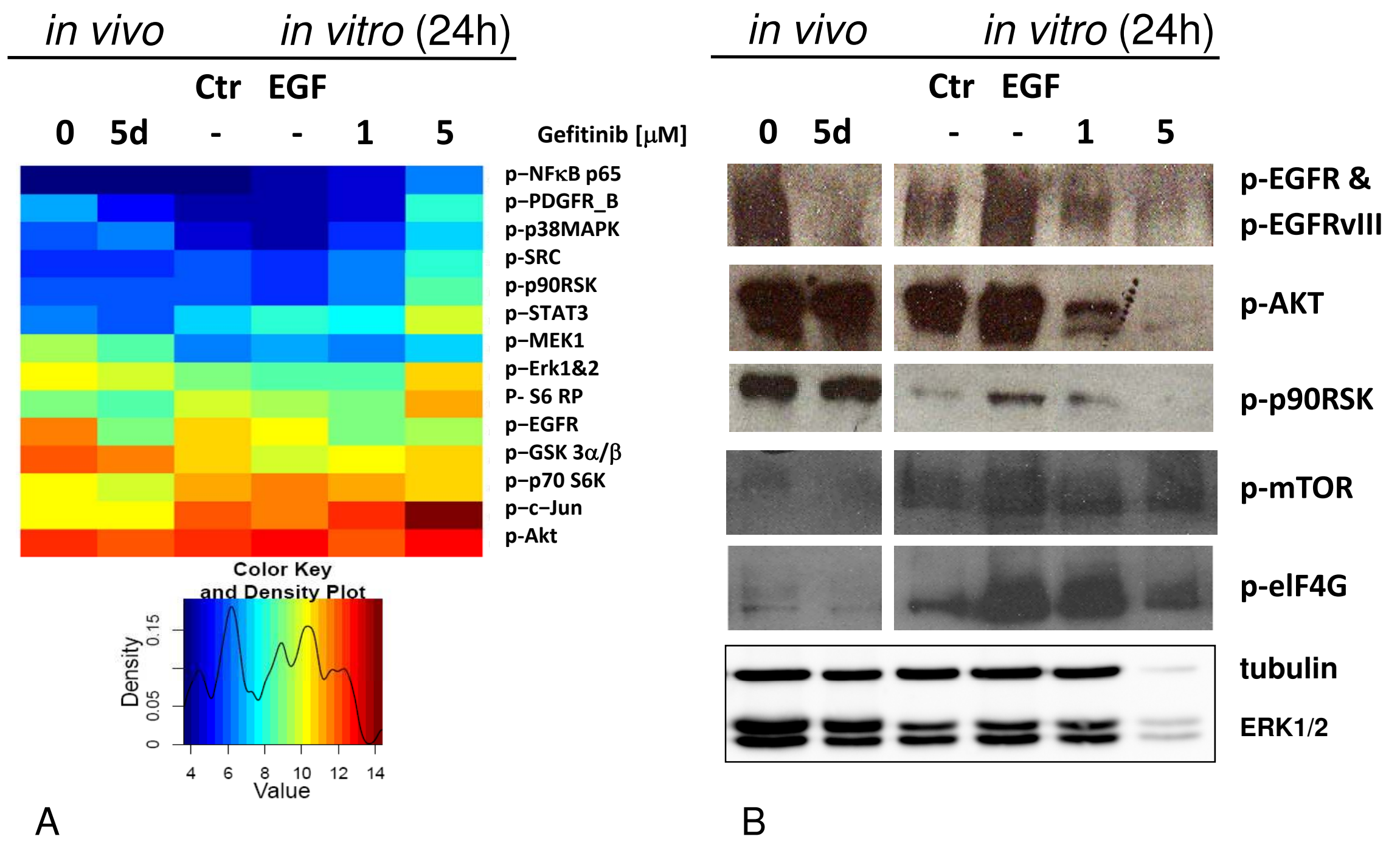

Fig. 5 
Supplementary Info

Hegi et al. 


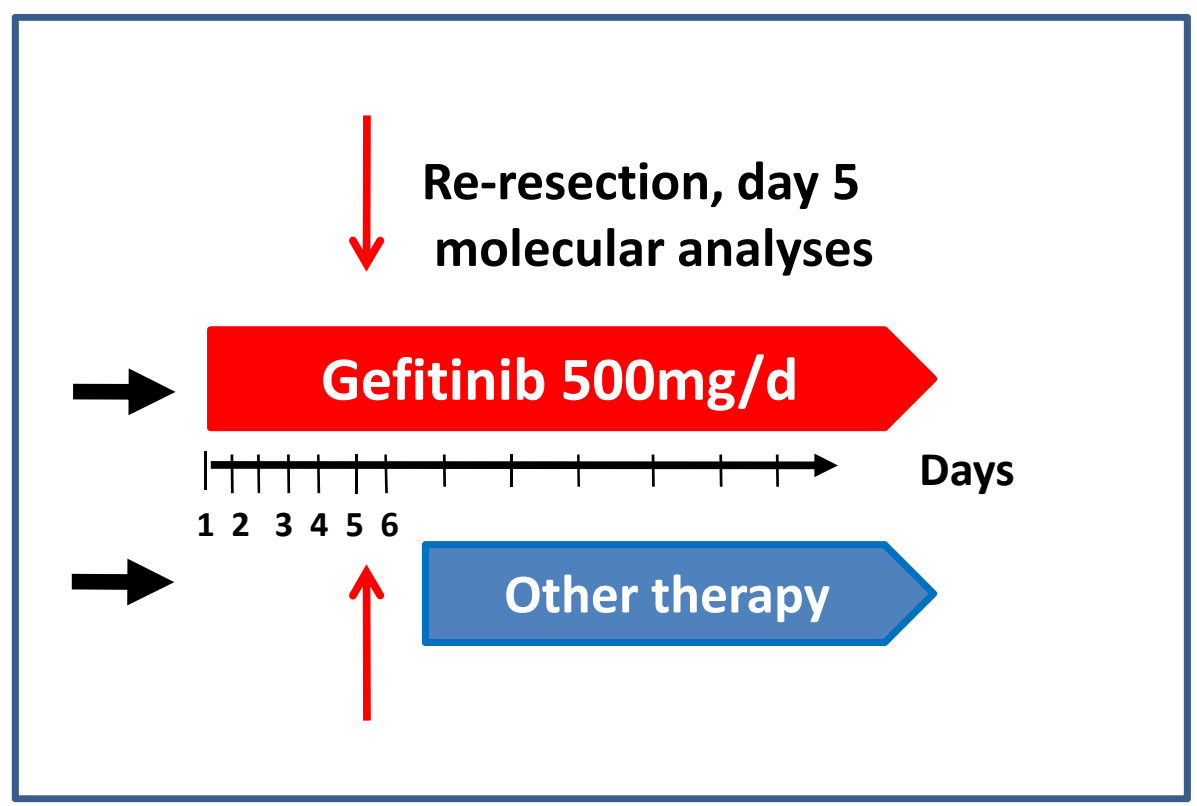

Fig. S1 
Fig. S2
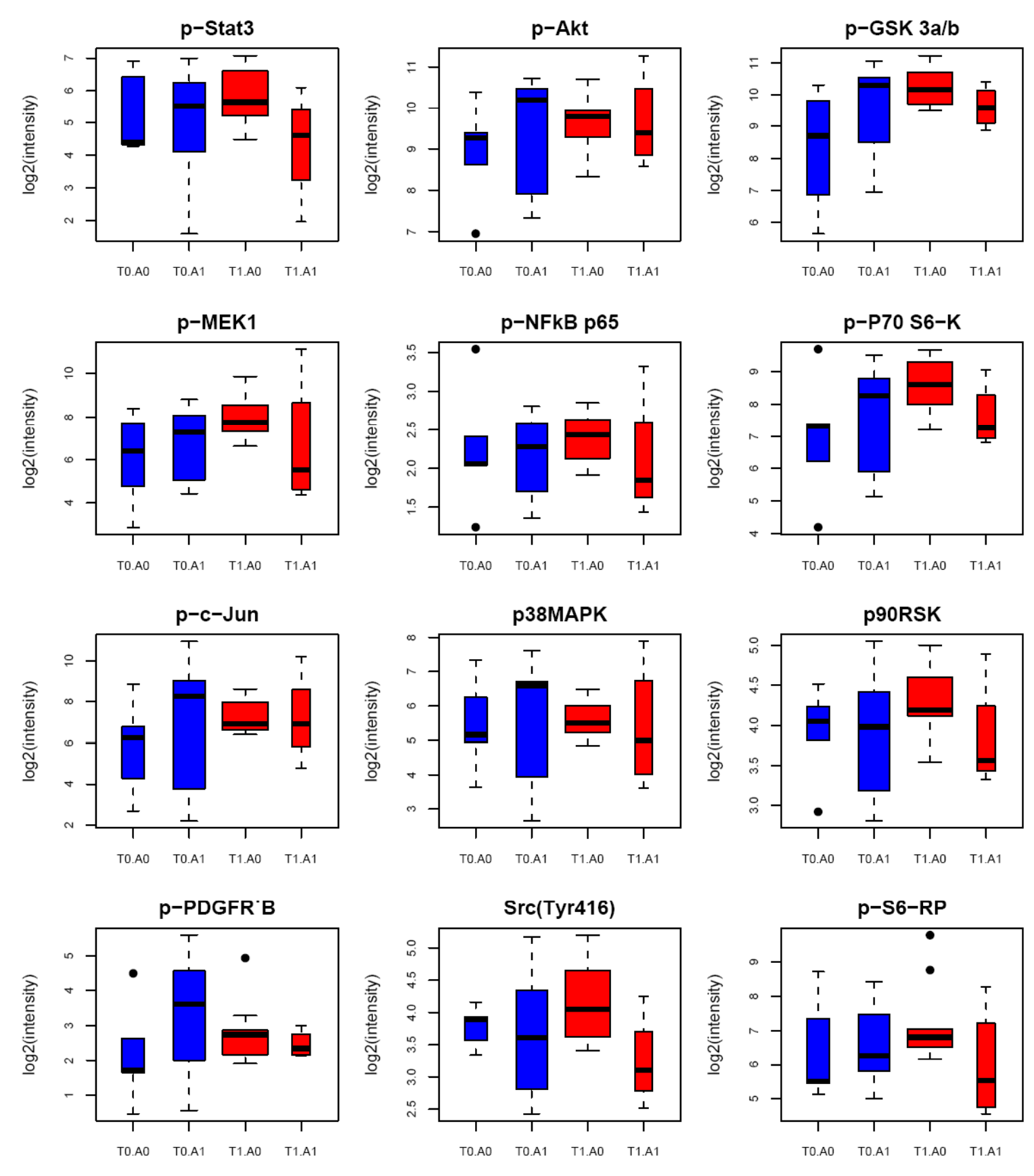


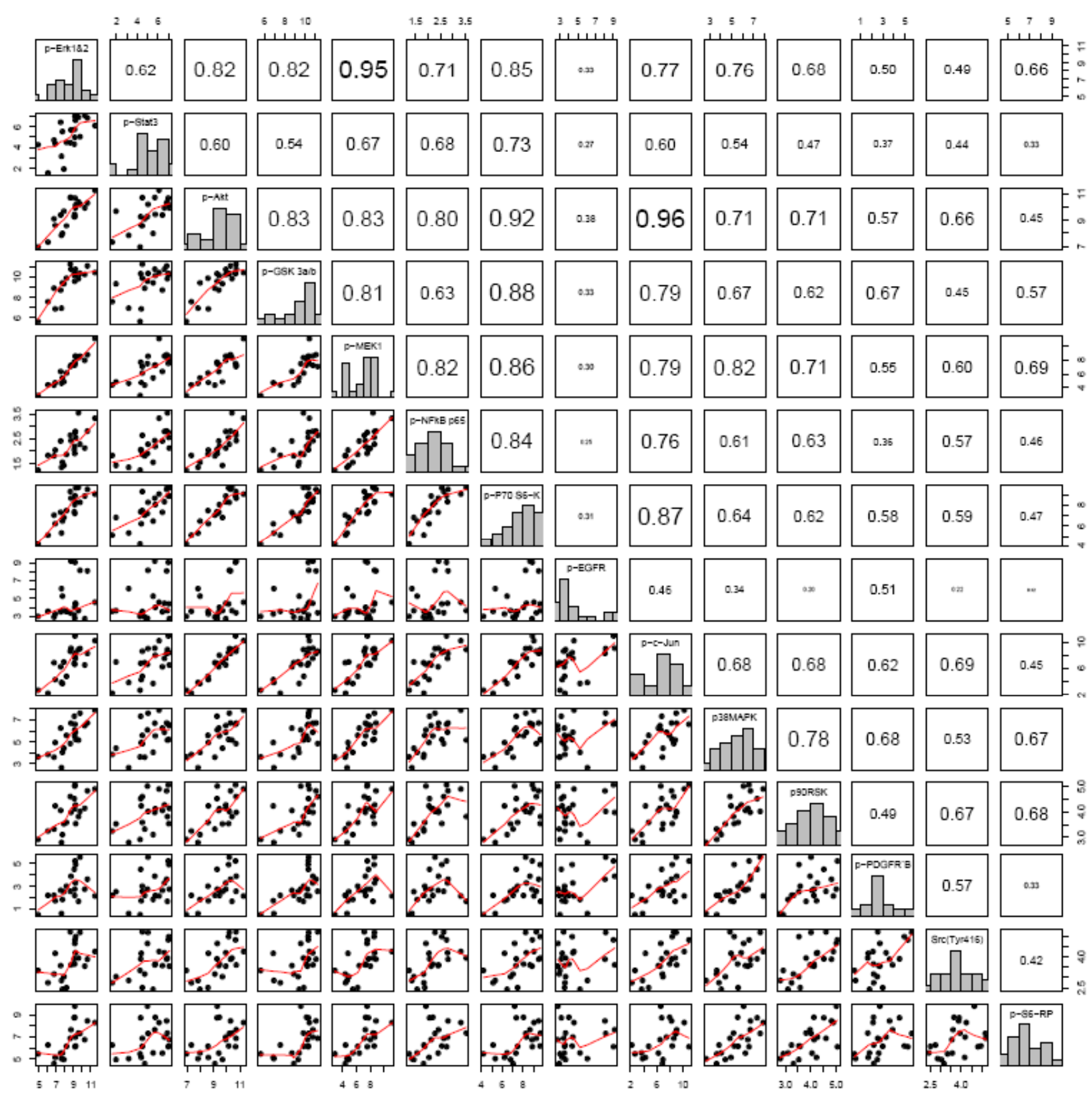

Fig S. 3 


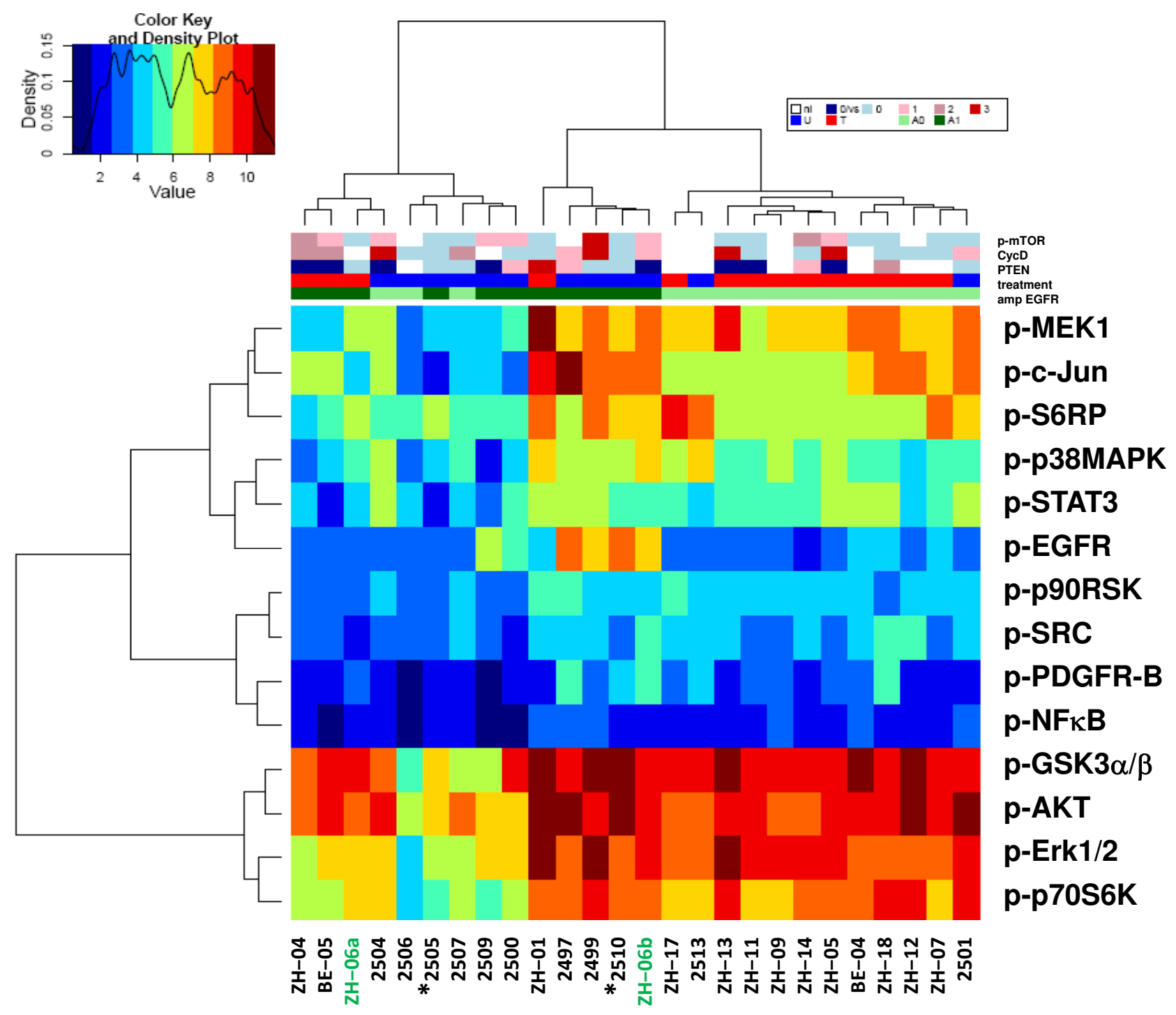

Fig. S4

Glioblastoma 
Supplemental data, Hegi et al.

Figure S1. Study outline. Patients selected for re-operation of a recurrent glioblastoma were enrolled in this phase II trial and were treated at least 5 days with $500 \mathrm{mg}$ gefitinib prior to surgery, followed by post-operative daily use, continuously until tumor progression or occurrence of intolerable side effects. Resected tumor tissue was collected for translational research. Control tissues were obtained from reresection of glioblastoma of patients treated outside clinical trials.

Figure S2. Effect of gefitinib on EGFR pathway signaling transductors. Fourteen signaling transductors of the EGFR pathway were measured by bioplex technology in tumor tissues under gefitinib treatment ( $\mathrm{T} 1$, red) or controls (T0, blue). The log2intensities of the phopsho-proteins, normalized by tot-Erk-1/2, are represented by box plots, stratified by the EGFR amplification status (A1, amplified; A0, not amplified).

Figure S3. Pairwise comparisons of phospho-proteins. A matrix of scatterplots of the bioplex values ( $n=26$ tumor samples, table normalized by $t-E r k 1 / 2)$ is shown. The lower triangle contains the cloud point representation between each variable (the loess smoothed curve is depicted in red). The upper triangle contains the values of Pearson correlation coefficients. The diagonal contains the histogram of each phosphor-protein.

Figure S4. Pattern of EGFR pathway activation in glioblastoma. The heatmap clusters the samples and phospho-proteins by similarity based on ward classification and euclidean distance. The dataset is normalized by total-Erk1/2, but neither scaled 
nor centered. Tumors with EGFR amplification are marked in green. Tumors under gefitinib treatment are indicated in red (T1), and blue for the controls (T0). This representation visualizes the fact that the amplitude varies a lot among the different phospho-proteins, e.g. between pEGFR and p-PDGFR-B. All labels are described in Fig. 4B. 
Table S1 Association of EGFR pathway signal transduction with gefitinib treatment

\begin{tabular}{|c|c|c|c|c|c|c|}
\hline \multirow[b]{2}{*}{ P-proteins } & \multicolumn{3}{|c|}{ Association with gefitinib treatment } & \multicolumn{3}{|c|}{$\begin{array}{l}\text { Associaton with gefitinib treatment } \\
\text { stratified for EGFR status }\end{array}$} \\
\hline & statistic. wilcox & p.value. wilcox & p.adj.wilcox & statistic.kruskal* & p.value.kruskal & p.adj.kruskal \\
\hline p-p38MAPK & 88 & 0.860 & 1.000 & 0.233 & 0.972 & 1.000 \\
\hline p-p90RSK & 60 & 0.231 & 1.000 & 3.468 & 0.325 & 1.000 \\
\hline p-Akt & 72 & 0.560 & 1.000 & 1.039 & 0.792 & 1.000 \\
\hline p-c-Jun & 70 & 0.494 & 1.000 & 1.637 & 0.651 & 1.000 \\
\hline p-EGFR & 122 & 0.053 & 0.632 & 12.358 & 0.006 & 0.075 \\
\hline p-Erk1\&2 & 49 & 0.076 & 0.908 & 6.157 & 0.104 & 1.000 \\
\hline p-GSK 3a/b & 63 & 0.297 & 1.000 & 4.464 & 0.216 & 1.000 \\
\hline p-MEK1 & 62 & 0.274 & 1.000 & 3.516 & 0.319 & 1.000 \\
\hline p-NFkB p65 & 73 & 0.595 & 1.000 & 2.085 & 0.555 & 1.000 \\
\hline p-P70 S6-K & 57 & 0.176 & 1.000 & 4.035 & 0.258 & 1.000 \\
\hline$p-S 6-R P$ & 73 & 0.595 & 1.000 & 3.614 & 0.306 & 1.000 \\
\hline p-Stat3 & 71 & 0.527 & 1.000 & 2.870 & 0.412 & 1.000 \\
\hline${ }^{* *} p-\operatorname{Src}(\operatorname{Tyr} 416)$ & 70 & 0.494 & - & 4.425 & 0.219 & - \\
\hline${ }^{* *} p-P D G F R \_B$ & 78 & 0.781 & - & 2.635 & 0.451 & - \\
\hline
\end{tabular}

${ }^{*}$ degree of freedom $=3$

**not considered as downstream EGFR pathway constituent, not included for Bonferroni correction 